\title{
ANALYSIS OF THE NOISE PARAMETERS AND ATTITUDE ALIGNMENT ACCURACY OF INS CONDUCTED WITH THE USE OF MEMS - BASED INTEGRATED NAVIGATION SYSTEM
}

\author{
Dariusz TOMASZEWSKI ${ }^{1{ }^{*} *}$, Jacek RAPIŃSKI ${ }^{1)}$ and Michał ŚMIEJA ${ }^{2)}$ \\ 1) Institute of Geodesy, University of Warmia and Mazury in Olcztyn, Poland \\ 2) Chair of Mechatronics, University of Warmia and Mazury in Olsztyn, Poland \\ *Corresponding author's e-mail: dariusz.tomaszewski@uwm.edu.pl
}

\section{ARTICLE INFO}

Article history

Received 4 February 2015

Accepted 16 April 2015

Available online 27 May 2015

Keywords:

INS alignment

GPS/INS integration

ARW

VRW

Allan Variance

\section{ABSTRACT}

Nowadays, along with the advancement of technology, the dynamic development of miniaturization in electronics can be noticed. Currently produced micro gyroscopes, accelerometers, magnetometers and GPS receivers have accuracy performance impossible to achieve few years ago. Thanks to this development, it is possible to build mobile integrated navigation systems based on satellite (GNSS) and inertial observations (IMU). Such method of navigation consists of several steps. The first stage is the determination of initial orientation of inertial measurement unit, called INS alignment. During this process, on the basis of acceleration and the angular velocity readings, values of Euler angles (pitch, roll, yaw) are calculated allowing for unambiguous orientation of the sensor coordinate system relative to external coordinate system. The following study presents the tests of accuracy of alignment conducted with the use of self - build GPS/INS integrated system.

\section{INTRODUCTION}

Along with the development of electronics one can notice that in present times a significant amount of equipment accompanies people in everyday life. Many of these devices (mobile phones, tablets) were designed thanks to miniaturization in electronics. This trend is also noticeable in the construction of miniaturized, mobile sensors such as gyroscopes, accelerometers or magnetometers. Devices of these type are called MEMS (Micro electro-mechanical systems). At the beginning these sensors had a very low accuracy which limited the possibilities of their use in more advanced applications. In currently produced devices, the accuracy have been significantly improved and it is now possible to find many applications that previously were not available for mobile devices.

One of these applications is to support the GPS satellite navigation using a set of inertial navigation sensors, called inertial measurement unit (IMU). After the implementation of navigational algorithms, an IMU module becomes Inertial Navigation System (INS). Integration of these two measurement systems is performed in order to increase the frequency of received coordinates and improve the quality of the results, obtained during a limited visibility of GPS satellites.

The IMU unit usually consists of three gyroscopes and three accelerometers. This set is sometimes accompanied by a magnetometer or other sensor in order to increase the accuracy of obtained results. Installed sensors are placed perpendicular to each other, thereby ensuring the possibility to perform measurements in three directions. Accelerometers placed in the IMU module measure linear acceleration in three mutually orthogonal direction. Simultaneously gyroscopes measure the angular rates in three mutually orthogonal directions. The axes of two triads are parallel, shearing the same origin. Thanks to, the set of sensors defines the axis of IMU body coordinate system also called the body frame (Figure 1; Foxlin, 2005).

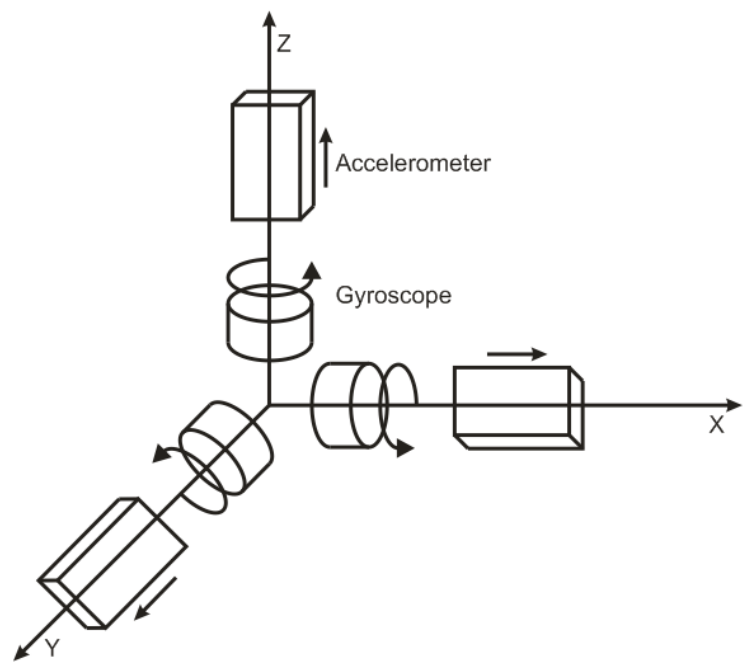

Fig. 1 The body frame of a typical inertial measurement unit (IMU). 


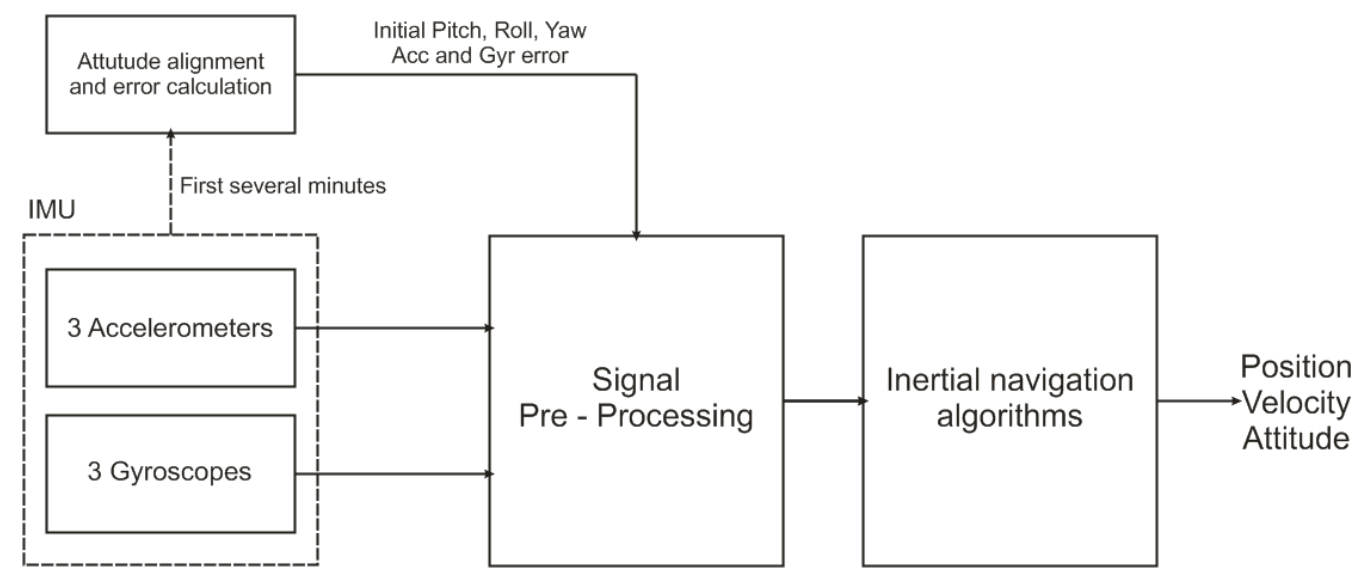

Fig. 2 The concept of inertial navigation.

Inertial navigation is the process in which through appropriate calculations made on the IMU data, one can determine the position, velocity and attitude. This process is complex and consists of several stages. Before starting of navigation it is necessary to know the initial orientation of the IMU unit, with respect to the external coordinate system (eg. WGS 84). This stage is called the attitude alignment and it is very important for the accuracy of results received during further navigation. At the same time errors of the accelerometers and gyroscopes readings are calculated. The time required to perform attitude alignment with error calculation depends on the used method and the accuracy of the sensors and can take from 2 to 10 minutes. During further navigation all the measurement data received from IMU are processed with the use of values calculated during initial alignment. Subsequently, by double integration of processed accelerations, the values of velocity and position are calculated. The attitude of the measurement unit, is updated with the use of gyroscope readings. Diagram showing the concept of inertial navigation is presented in Figure 2 (Farrell, 2008).

As it was mentioned before, the attitude alignment is the process in which the initial orientation of IMU unit is determined. The method most commonly used for land navigation is calculation of the Euler angles (pitch, roll and yaw) on the basis of gravity and rotation measurements gathered during a few minutes in which the INS module remains stationary. Calculated pitch, roll and yaw angles are defined as rotation of the body of IMU unit with respect to external coordinate system (Figure 3). Precise determination of Euler angles allows to determine the impact of the gravity acceleration and Corriolis force on sensors readings (Noureldin et al., 2013).

The figure above shows that the pitch and roll angles are responsible for the $\mathrm{y}$ and $\mathrm{x}$ rotations

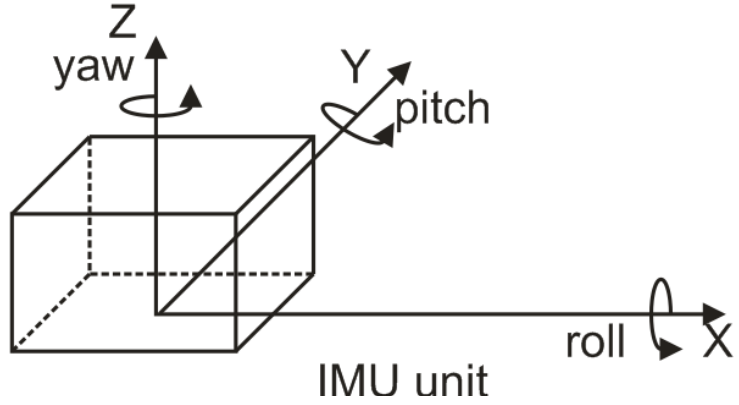

Fig. 3 Presentation of the Euler angles.

respectively and yaw angle corresponds to the rotation around $\mathrm{z}$ axis of body frame.

Attitude alignment involves two steps. First the IMU body is leveled by initializing the pitch and roll angles with the use of gravity acceleration measurements registered by accelerometers. This step is called accelerometer leveling. The second step of attitude alignment is gyro-compassing. This step provides an initial value of heading, alternatively known as the yaw angle or azimuth. Gyro-compassing is calculated on the basis of gyroscope readings. Accuracy of calculated angles depends directly on the accuracy of the sensors used.

The main goal of this paper is to test the accuracy of the IMU module mounted inside the selfbuild GPS/INS system. This system consists of ADIS 16354 MEMS IMU and uBlox LEA6T GPS receiver. The ADIS16354 three-axis inertial sensor is a measurement module, consisting of three accelerometers and gyroscopes. This IMU unit has the ability to perform measurements at $1 \mathrm{~Hz}-350 \mathrm{~Hz}$ sampling rate with 14 bit resolution (Analog Devices). Accelerometer mounted inside the IMU module makes it possible to perform readings with dynamic range $\pm 1.7 \mathrm{~g}$. The gyroscope installed within ADIS 16354 measures angular rate with dynamic ranges 


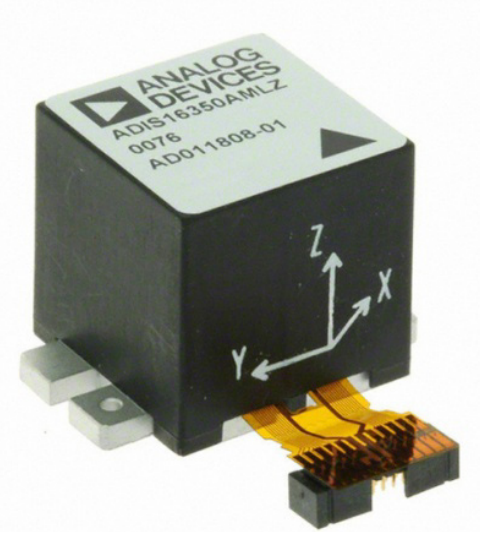

Fig. 4 ADIS 16354 IMU measurement unit.

from $\pm 75 \%$ to $\pm 300 \%$ with smallest possible sensitivity of $0.01832 \% / \mathrm{LSB}$. The ADIS 16354 is presented in Figure 4.

In the study, the values of basic noise parameters of the IMU sensors are determined. For the accelerometer, these parameters are velocity random walk and bias instability. Similarly, for gyro angular random walk and bias instability. Afterwards, the accuracy of attitude alignment conducted with the use of ADIS 16354 are determined experimentally.

\section{MEMS IMU ERRORS CHARACTERISTICS AND ALLAN VARIANCE}

Despite the reduction of MEMS IMU measurement errors, in recent years, these errors still significantly affects obtained results. These errors get more complex as the price of the sensor goes down. This limits the accuracy to which the observables can be measured (Titterton and Weston, 2009). The IMU errors are classified according to two broad categories of systematic and random errors.

Systematic errors are those which can be calibrated in laboratory conditions. The most common errors are (Noureldin et al., 2013):

- Constant bias - can be defined as an output of the sensor (accelerometer or gyroscope) while it is not affected by any external force or angular rate. When calculating unit the position, the value of constant bias is integrated twice causing an error in position to grow quadratically with time. This bias can be eliminated in laboratory by performing long term measurements while the sensor is not undergoing any acceleration or rotation.

- Scale factor error -is defined as the deviation of the input - output gradient from unity. The value of this error is proportional to the value of observables measured by the sensors.

- Scale factor sign asymmetry - is defined as error due to different scale factors for positive and negative measured values

- Dead zone - is the region between the input limit within witch variations in the input produce output changes of less $10 \%$ of those expected based on nominal scale factor.

- Non-orthogonality error - this error is generated during the manufacturing process of the sensors. It means that the axes of the coordinate system formed by the sensors are not perfectly perpendicular to each other.

- Misalignment error - this error is also due to defects in manufacturing of IMU. The result of this error is misaligning the sensitive axis of the inertial sensors relative to the body frame presented on the unit.

As it can be noticed, presented systematic errors are permanent. In order to eliminate them laboratory calibrations are carried out with the use of specialized equipment. Then the impact of these errors on recorded observables is compensated at the beginning of each measurement.

Simultaneously, the measurements made with the use of inertial sensors are affected by random errors, which, due to their unpredictability, have much more influence on the results. These errors are usually modelled stochastically in order to mitigate their effects. The most common errors are (Groves, 2008):

- Bias stability - a random change in bias over time. This change results from the instability of sensor measurement during single navigation and is also called bias drift. Bias is deterministic but drift is stochastic.

- Scale factor instability - a random change in scale factor. It is usually the result of temperature change. This bias is constant during single run but can change in subsequent navigation.

- White noise - an uncorrelated noise mostly caused by the power source of the sensor and temperature variations.

Value most commonly used to determine the randomness associated with inertial sensors is random walk. Usually, by that means, a direct impact of an uncorrelated noise on the calculated angles and velocities is presented by the manufacturers of inertial sensors. Random walk is produced by integrating inertial sensors white noise of which standard deviation grows proportionally to $\sqrt{t}$. Hence, white noise on the output of an gyroscope creates an angle random walk (ARW), specified with units $\% \sqrt{h}$. Depending on the sensor price, the value of ARW varies from $0.002 \% \sqrt{h}$, for navigation grade sensors, to several $\% \sqrt{h}$ for commercial grade sensor. For instance, if the value of ARW equals $0.4 \% \sqrt{h}$, this means that after one hour the standard deviation of orientation error will be 0.4 and after three hours 


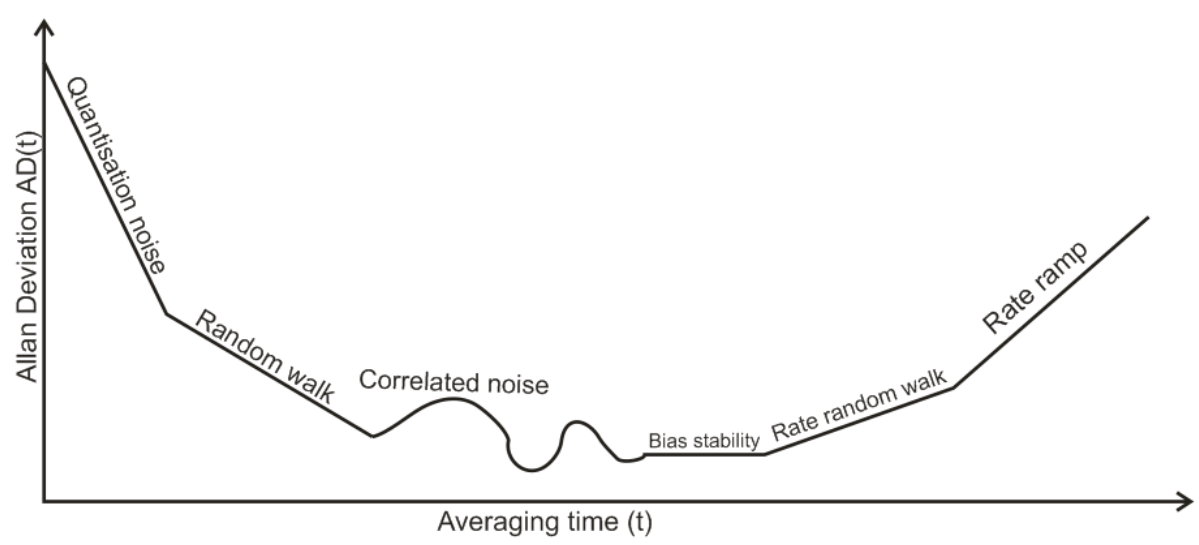

Fig. 5 Sample shape of logarithmic Allan Deviation graph.

$\sqrt{3} * 0.4=0.69^{\circ}$. Similarly, by integrating the white noise of an accelerometer, one gets the value known as velocity random walk (VRW), specified with $\mathrm{m} / \mathrm{s} / \sqrt{h}$ unit (Woodman, 2007).

The second parameter used for describing the value of sensor randomness is bias stability, also called flicker noise. This parameter is the most frequently used for the gyroscope accuracy determination. The bias fluctuations are usually modelled as a random walk. This error describes how the value of constant bias will change over specific period of time. Bias stability is usually specified with $\%$ or $\% / h$ units depending on the accuracy of the device (Titterton and Weston, 2009).

A method which is most commonly used for estimating the value of ARV, VRW, bias stability of MEMS IMU sensors is Allan Variance (Stockwell). This technique was originally designed for characterizing noise and stability in clock systems. However, it may be used for determining noise of any time correlated signal. The Allan Variance is calculated as a function of averaging time. Algorithm of Allan Variance is as follows(Allan, 1966):

1. Recording the data for a long period of time.

2. Recorded data are divided into parts of length equal to the selected value of averaging times $t$.

3. Data for each of the parts are averaged obtaining a list of averages $\left(a(t)_{1}, a(t)_{2}, \ldots, a(t)_{n}\right)$

4. The Allan Variance is given by:

$A V A R(t)=\frac{1}{2 \cdot(n-1)} \sum\left(a(t)_{i+1}-a(t)_{i}\right)^{2}$

Where:

$\mathrm{t}$ - averaging time,

$a(t)$ - one averaged part,

$\mathrm{n}$ - number of averaged parts (min 9 parts)

5. To define a characteristic of noise process, Allan Deviation is calculated and plotted as a function I logarithmic scale (Figure 4).
$A D(t)=\sqrt{A ́ V A R(t)}$

As it can be seen in Figure 5, different kind of process causes slopes with different gradients on the plotted function. The presence of various types on underlying noise appears in different regions of the value of ARN and VRN in s obtained at averaging time at $t=1$ s (Han et al., 2009). The value of bias stability is the flat region at the minimum of the Allan Deviation curve (Wang and Williams, 2008).

\section{ADIS 16354 RANDOM ERROR ANALYSIS}

In order to determine the values of velocity random walk, angle random walk and bias instability for ADIS accelerometer and gyroscope three $24 \mathrm{~h}$ measurement sessions were performed. During these sessions, the IMU unit was leveled and remained stationary with one axis pointing up. ADIS performed readings with $100 \mathrm{~Hz}$ frequency. The Allan Variance technique was applied to recorded data. Figure 6 depicts the shape of Allan Deviation graphs for accelerometer readings.

As it can be noticed, three accelerometers mounted within ADIS IMU have similar performance. The numerical values of VRW and accelerometers bias instability are presented in Table 1.

The value of bias stability for $\mathrm{Z}$ axis is two times lower than the for $\mathrm{X}$ and $\mathrm{Y}$ axes. This result may suggest that the measurements for the $\mathrm{Z}$-axis was incorrectly made. Therefore, the experiment was repeated to ensure that the result is true. Obtained results indicate that ADIS 16354 has a middle class accelerometer build-in (Vukmirica et al., 2010). The value of VRW found on ADIS data sheet is $0.135 \mathrm{~m} / \mathrm{s} / \sqrt{h}$. However, the results of the study shows that the performance of Velocity Random Walk is twice worst. The similar analysis was performed for the gyroscope readings. Results for gyroscope readings are presented in Figure 7.

As it can be noticed, the noise performance for all three axes of gyroscope is similar. The values of 

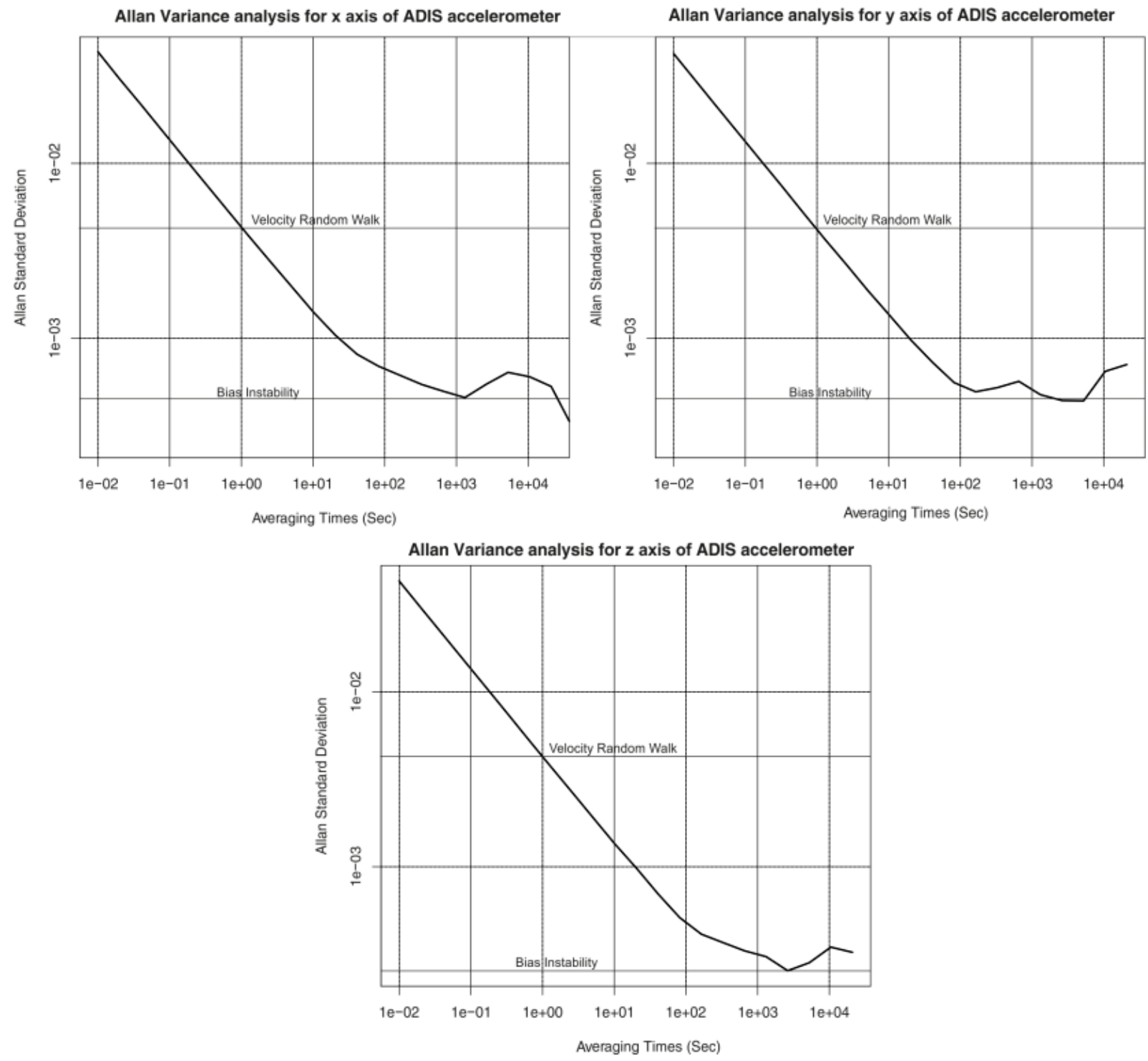

Fig. 6 Allan Variance analysis of accelerometer installed within ADIS 16354 IMU module.

Table 1 Accelerometer noise measurements.

\begin{tabular}{lcl}
\hline & Velocity Random Walk & Bias Stability \\
\hline X Axis & $0.23 \mathrm{~m} / \mathrm{s} / \sqrt{h}$ & $0.038 \mathrm{~m} / \mathrm{s}^{2}$ \\
Y Axis & $0.27 \mathrm{~m} / \mathrm{s} / \sqrt{h}$ & $0.034 \mathrm{~m} / \mathrm{s}^{2}$ \\
Z Axis & $0.27 \mathrm{~m} / \mathrm{s} / \sqrt{h}$ & $0.017 \mathrm{~m} / \mathrm{s}^{2}$ \\
\hline
\end{tabular}

ARW and bias stability for $\mathrm{Z}$ axis are marginally smaller, however, this difference is relatively small. Performed analysis implies that the variations in orientation calculated on a basis of measurements recorded by installed gyroscope are characteristic for middle-class devices. The values of AWR and BS for ADIS gyro presented in data sheet are respectively $0.015 \%$ and $42 \% \sqrt{h}$, if one were to compare them with the results obtained from the study it can be concluded that the values are similar The values of ARW indicate that this is a commercial grade gyro (Petkov and Slavov, 2010).

\section{THE ATTITUDE ALIGNMENT}

The attitude alignment consists of two stages. In the first one, called accelerometer leveling, the 

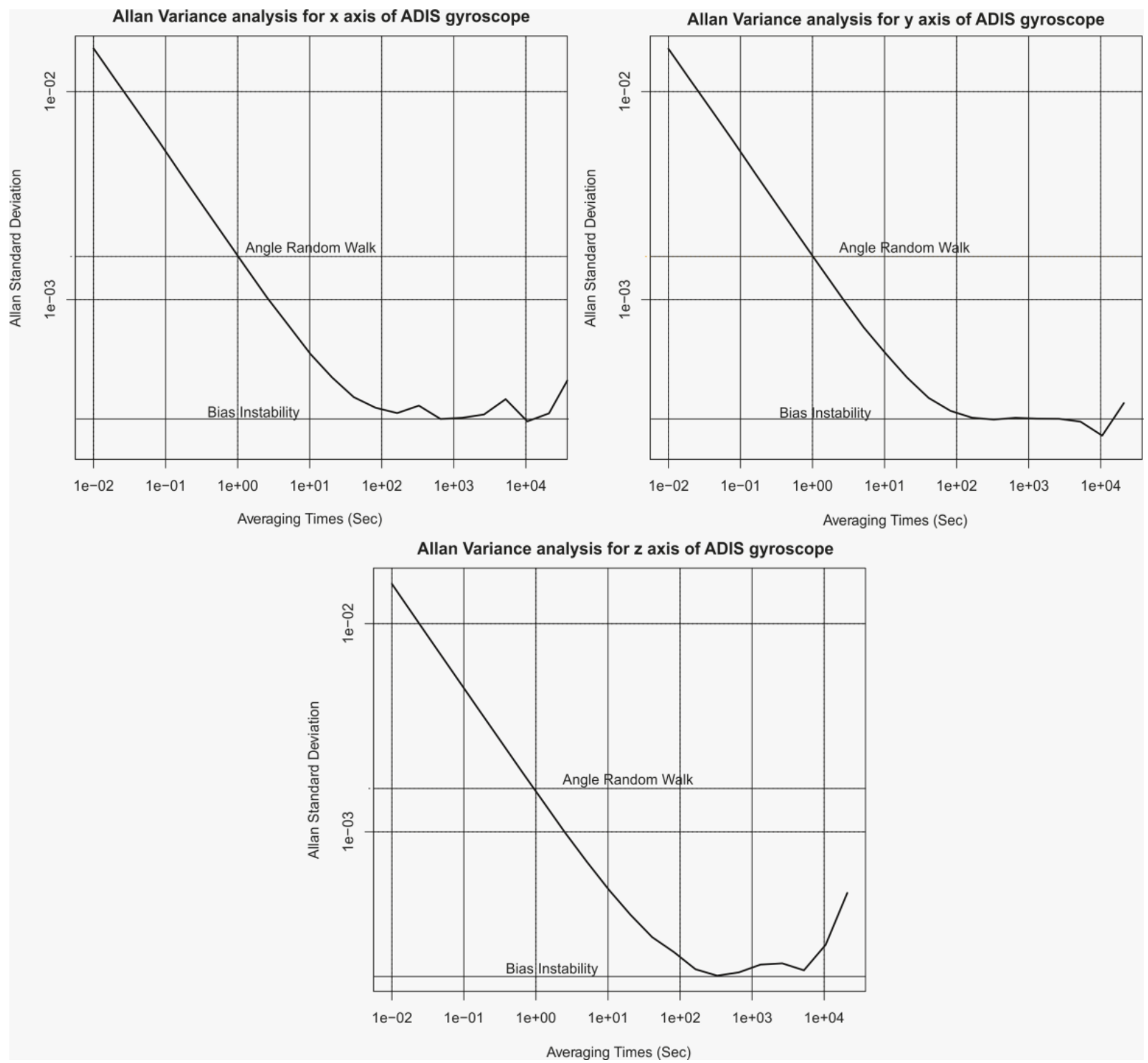

Fig. 7 Allan Variance analysis of gyroscope installed within ADIS 16354 IMU module.

Table 2 Gyroscope noise measurements.

\begin{tabular}{lcc}
\hline & Angle Random Walk & Bias Stability \\
\hline X Axis & $5.93 \% / \sqrt{h}$ & $0.015 \%$ \\
Y Axis & $5.92 \% / \sqrt{h}$ & $0.013 \%$ \\
$Z$ Axis & $5.68 \% / \sqrt{h}$ & $0.012 \%$ \\
\hline
\end{tabular}

gravitational acceleration is measured by a stationary IMU unit. During this time accelerometers measure the components of the reaction to gravity due to the pitch and roll angles. The values observed by the accelerometers on the axes $\mathrm{X}$ and $\mathrm{Y}$ indicate a tilt with respect to the horizontal plane. The measurements performed by an accelerometer can be expressed as follows (Noureldin et al. 2013):

$$
a=C_{i}^{b}(g)=\left(C_{b}^{i}\right)^{T}\left(-g_{a}\right)
$$

where:

a is a vector of accelerations measured by the IMU device 
$a=\left[\begin{array}{l}a_{x} \\ a_{y} \\ a_{z}\end{array}\right]$

$a_{x}, a_{y}, a_{z}-$ measurements made by respective axes of accelerometer

$C_{b}^{i}$ is a rotation matrix between the body frame of the inertial measurement unit and local level frame

$C_{b}^{i}=\left|\begin{array}{ccc}\cos \psi \cos \phi-\sin \psi \sin \Theta \sin \phi & -\sin \psi \cos \Theta & \cos \psi \sin \phi+\sin \psi \sin \Theta \cos \phi \\ \sin \psi \cos \phi+\cos \psi \sin \Theta \sin \phi & \cos \psi \cos \Theta & \sin \psi \sin \phi-\cos \psi \sin \Theta \cos \phi \\ -\cos \Theta \sin \phi & \sin \Theta & \cos \Theta \cos \phi\end{array}\right|$

$\psi, \Theta, \phi$ - yaw, pitch, roll angles respectively.

$\mathrm{g}$ is theoretical gravity vector.

$g_{a}=\left[\begin{array}{c}0 \\ 0 \\ -g\end{array}\right]$

$g$ - is the gravitational acceleration, (mean value for Earth $9.81\left(\mathrm{~m} / \mathrm{s}^{2}\right)$ ).

Substituting these values to first equation gives:

$a=\left|\begin{array}{ccc}\cos \psi \cos \phi-\sin \psi \sin \Theta \sin \phi & \sin \psi \cos \phi+\cos \psi \sin \Theta \sin \phi & -\cos \Theta \sin \phi \\ -\sin \psi \cos \Theta & \cos \psi \cos \Theta & \sin \Theta \\ \cos \psi \sin \phi+\sin \psi \sin \Theta \cos \phi & \sin \psi \sin \phi-\cos \psi \sin \Theta \cos \phi & \cos \Theta \cos \phi\end{array}\right|\left[\begin{array}{l}0 \\ 0 \\ g\end{array}\right]$

$\left[\begin{array}{l}a_{x} \\ a_{y} \\ a_{z}\end{array}\right]=\left[\begin{array}{c}g \cos \Theta \sin \phi \\ g \sin \Theta \\ g \cos \Theta \cos \phi\end{array}\right]$

and finally the values of pitch and roll angles are calculated as:

$\Theta=\tan ^{-1}\left(\frac{a_{x}}{\sqrt{a_{x}^{2}+a_{z}^{2}}}\right)$
$\phi=\tan ^{-1}\left(\frac{-a_{x}}{a_{z}}\right)$

In case of roll angle the four-quadrant arctangent function must be used.

The second stage of attitude alignment is gyrocomapssing. During this step, the Earth's rotation rate is measured. While unit remains stationary, the gyroscope measure only the rotation of the body frame with respect to the Earth frame (E - frame). Then, the gyroscope measurements fulfil the following equation (Groves, 2008).

$\omega_{i b}^{b}=C_{l}^{b} C_{e}^{l} \omega_{i e}^{e}=\left(C_{b}^{l}\right)^{T}\left(C_{l}^{e}\right)^{T} \omega_{i e}^{e}$

Where $\omega_{i b}^{b}$ is the vector of angular velocities measured by gyroscope.

$\omega_{i b}^{b}=\left[\begin{array}{c}\omega_{x} \\ \omega_{y} \\ \omega_{z}\end{array}\right]$

$\omega_{x}, \omega_{y}, \omega_{z}$ measurements made by around axes of gyroscope 
The $R_{b}^{l}$ rotation matrix was presented in previous stage of attitude alignment. $C_{i}^{e}$ is a rotation matrix between local level frame and Earth frame.

$C_{l}^{e}=\left|\begin{array}{ccc}-\sin \lambda & -\sin \varphi \cos \lambda & \cos \varphi \cos \lambda \\ \cos \lambda & -\sin \varphi \sin \lambda & \cos \varphi \sin \lambda \\ 0 & \cos \varphi & \sin \varphi\end{array}\right|$

$\varphi, \lambda$ - latitude and longitude of the IMU unit

$\omega_{i e}^{e}$ is the Earth's rotation rate vector.

$\omega_{i e}^{e}=\left[\begin{array}{c}0 \\ 0 \\ \omega_{e}\end{array}\right]$

$\omega_{e}-$ Earth's rotation rate equal $7.292115 .10^{-5} \mathrm{rad} / \mathrm{s}$.

Substituting these values to the first equation gives.

$\omega_{i b}^{b}=\left|\begin{array}{ccc}\cos \psi \cos \phi-\sin \psi \sin \Theta \sin \phi & \sin \psi \cos \phi+\cos \psi \cos \Theta \sin \phi & -\cos \Theta \sin \phi \\ -\sin \psi \cos \Theta & \cos \psi \cos \Theta & \sin \Theta \\ \cos \psi \sin \phi+\sin \psi \sin \Theta \cos \phi & \sin \psi \sin \phi-\cos \psi \sin \Theta \cos \phi & \cos \Theta \cos \phi\end{array}\right|$

$\left[\begin{array}{c}\omega_{x} \\ \omega_{y} \\ \omega_{z}\end{array}\right]=\left[\begin{array}{c}\{\sin \psi \cos \phi+\cos \psi \sin \Theta \sin \phi\} \omega_{e} \cos \varphi-\omega_{e} \cos \Theta \sin \phi \sin \varphi \\ \omega_{e} \cos \varphi \cos \psi \sin \Theta+\omega_{e} \sin \varphi \sin \Theta \\ \{\sin \psi \sin \phi-\cos \psi \cos \Theta\} \omega_{e} \cos \varphi+\omega_{e} \cos \Theta \cos \phi \sin \varphi\end{array}\right]$

The values of pitch and roll angles are known from accelerometer levelling. The yaw angle is only unknown in these set of equations. After performing a set of transformations one can obtain the final value of yaw angle equal:

$\psi=\tan ^{-1}\left[\frac{\omega_{x} \cos \phi+\omega_{z} \sin \phi}{\omega_{y} \cos \Theta+\omega_{x} \sin \Theta \sin \phi-\omega_{z} \cos \phi \sin \Theta}\right]$

In the case of yaw angle the four-quadrant arctangent function must be used.

As it was noted before in the case of gyrocomapssing the Earth's rotation rate have to be measured. This value is very small and equals about $7 \times 10^{-5} \mathrm{rad} / \mathrm{s}$, so to obtain $1 \mathrm{mrad}$ yaw initialization at the equator the gyro must be accurate to around $7 \times 10^{-8} \mathrm{rad} / \mathrm{s}$. This value indicates that only gyroscopes with bias stability less than $5 \% / h$ are capable of gyrocomassing. For low grade IMU, the value of heading is calculated on the basis of compass, magnetometer or GPS in the case of integrated systems.

\section{THE TEST OF ATTITUDE ALIGNMENT ACCURACY CONDUCTED WITH ADIS 16354 IMU. ACCELEROMETER LEVELING}

To perform the accuracy test of the pitch and roll calculation, with the use of ADIS 16354 IMU module, eight 360 second measurement sessions were made. During each session, IMU was tilted by a predetermined value of pitch or roll. To ensure accurate determination of the measured angles, the module was placed on the telescope of the Leica TS30 total station (Figure 8.).

The first four sessions were made for the pitch angle. During the measurements, the IMU unit was subsequently tilted by $0^{\circ}, 10^{\circ},-10^{\circ},-20^{\circ}$. The accuracy of simulated inclination angles was $0.00004^{\circ}$. The value of the pitch angle was calculated for every 10 second of conducted observations. The results of performed calculations are presented in the figures below.

As it can be noticed in Table 2 the value of standard deviation was calculated for each measurement. Afterwards, on the basis of conducted research, the mean value of standard deviation for pitch angle was calculated. The standard deviation of calculated angles is $0.0084^{\circ}$. Such an error in the initial orientation cause a deviation calculating the 


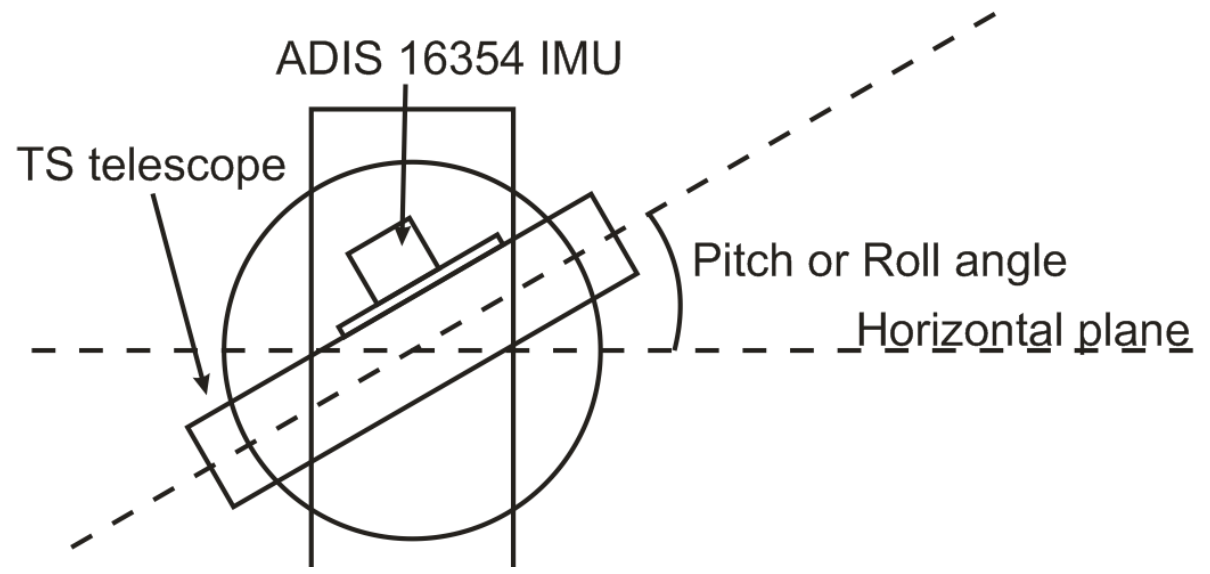

Fig. 8 Scheme of experiments equipment installation.

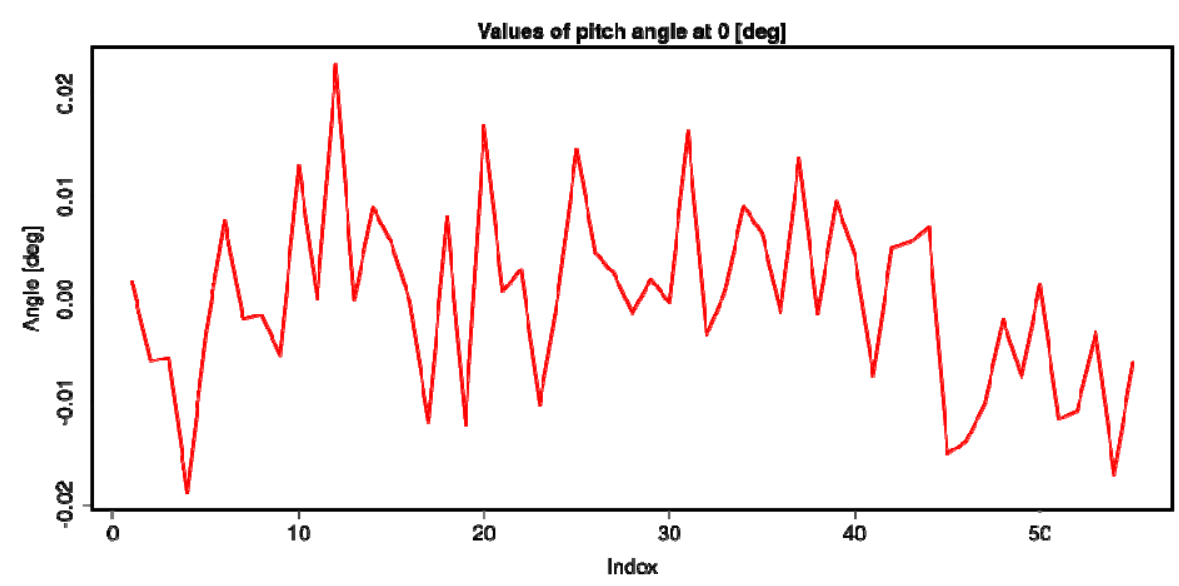

Fig. 9 Pitch values calculated for $0^{\circ}$ tilt.

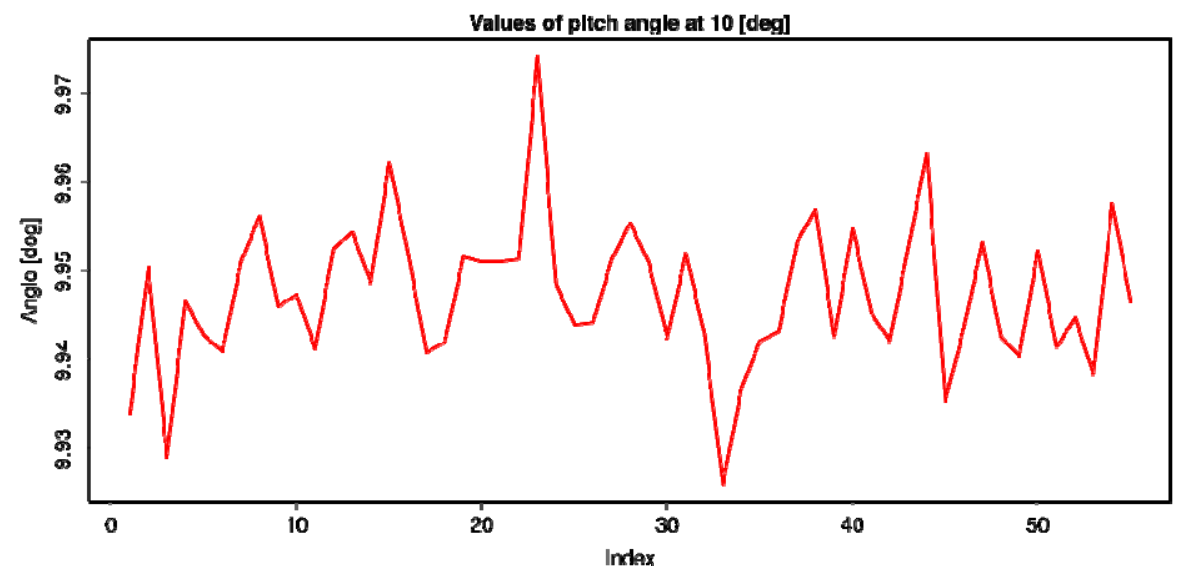

Fig. 10 Pitch values calculated for $10^{\circ}$ tilt. 


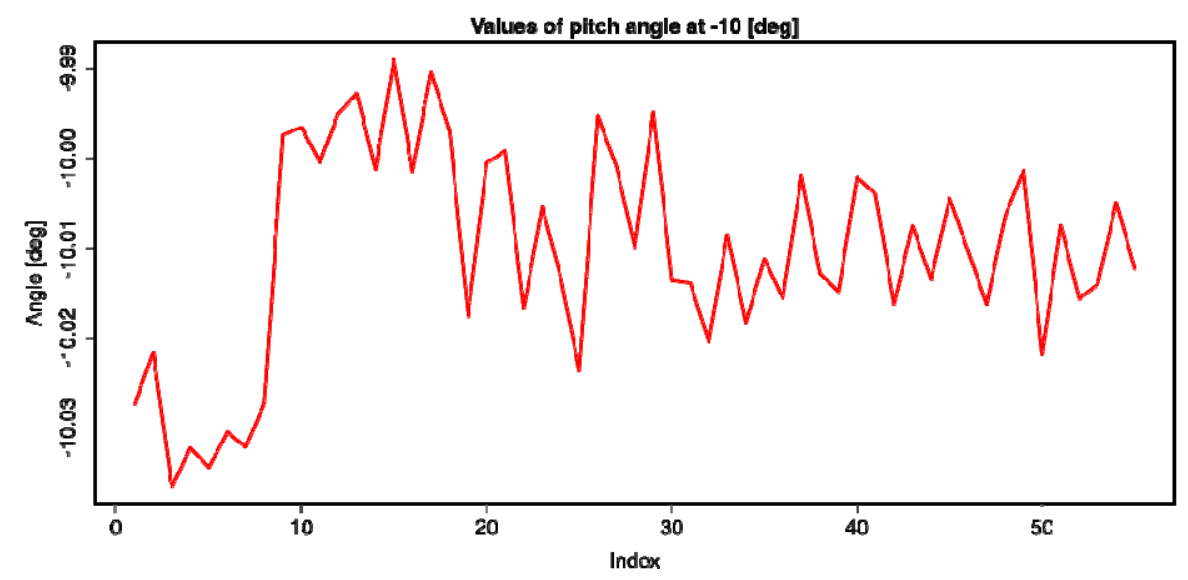

Fig. 11 Pitch values calculated for $-10^{\circ}$ tilt.

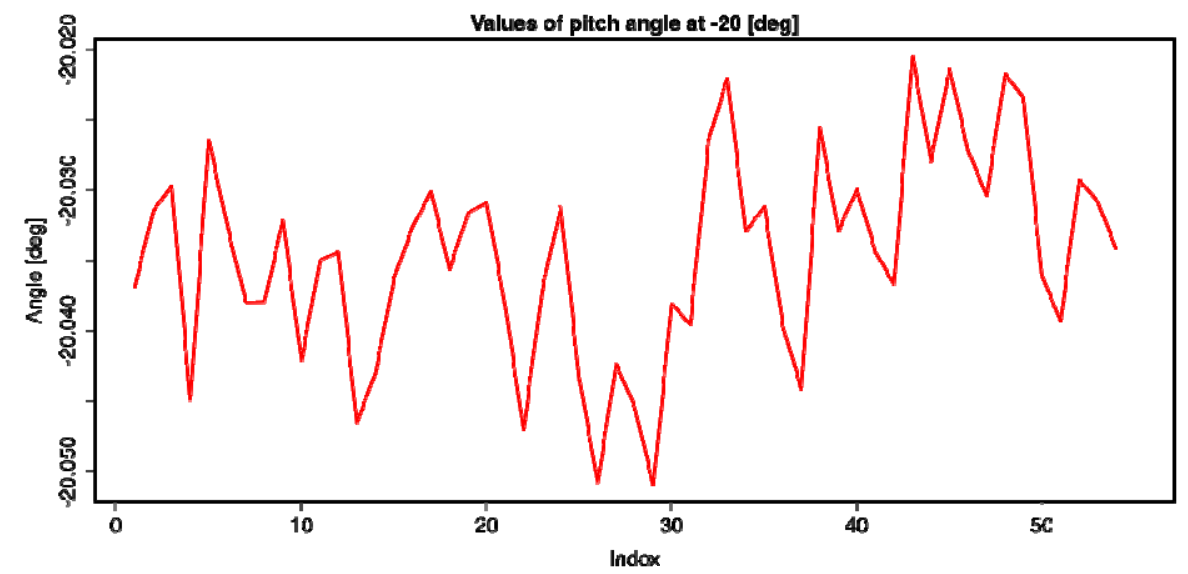

Fig. 12 Pitch values calculated for $-20^{\circ}$ tilt.

Table 2 Summary of conducted measurements.

\begin{tabular}{rrrrrc}
\hline Angle & mean & Min & Max & variance & $\begin{array}{c}\text { Standard } \\
\text { deviation }\end{array}$ \\
\hline $0^{\circ}$ & $0.000^{\circ}$ & $-0.019^{\circ}$ & $0.0228^{\circ}$ & $0.0000813^{\circ}$ & $0.0090^{\circ}$ \\
$10^{\circ}$ & $9.947^{\circ}$ & $9.926^{\circ}$ & $9.9740^{\circ}$ & $0.0000692^{\circ}$ & $0.0083^{\circ}$ \\
$-10^{\circ}$ & $-10.010^{\circ}$ & $-10.040^{\circ}$ & $-9.9890^{\circ}$ & $0.0000785^{\circ}$ & $0.0089^{\circ}$ \\
$-20^{\circ}$ & $-20.030^{\circ}$ & $-20.050^{\circ}$ & $20.0200^{\circ}$ & $0.0000543^{\circ}$ & $0.0073^{\circ}$ \\
\hline mean & & & $0.0000708^{\circ}$ & $0.0084^{\circ}$ \\
\hline
\end{tabular}

position equal $0.26 \mathrm{~m}$ after 60 seconds of measurement.

A similar study was performed to determine the error of roll angle calculation. Four sessions were made, during which the IMU unit was slanted by $0^{\circ},-10^{\circ},-20^{\circ},-30^{\circ}$. The roll value was calculated for every 10 second of conducted observation. The results of performed calculations are presented in the figures below.

The results showed similar performance for accelerometers installed on both axes. As it can be noticed in the table above the value of standard deviation was calculated for each measurement. Afterwards, as in case of pitch angle, the mean standard deviation for roll value is $0.0084^{\circ}$ causing an error in calculated position $0.26 \mathrm{~m}$ after $60 \mathrm{~s}$ of observation.

\section{GYROCOMPASSING}

As it was mentioned before, calculation of yaw angle on the basis of gyroscope measurements require the device with the value of bias stability lower then $5 \%$. On the b asis of conducted research the value of bias stability, for gyroscope installed on $\mathrm{z}$ axis of ADIS 16354 IMU unit, is $0.012 \%$ with gives $43.2 \%$. This means that after an hour of measurement the value of constant bias can change 


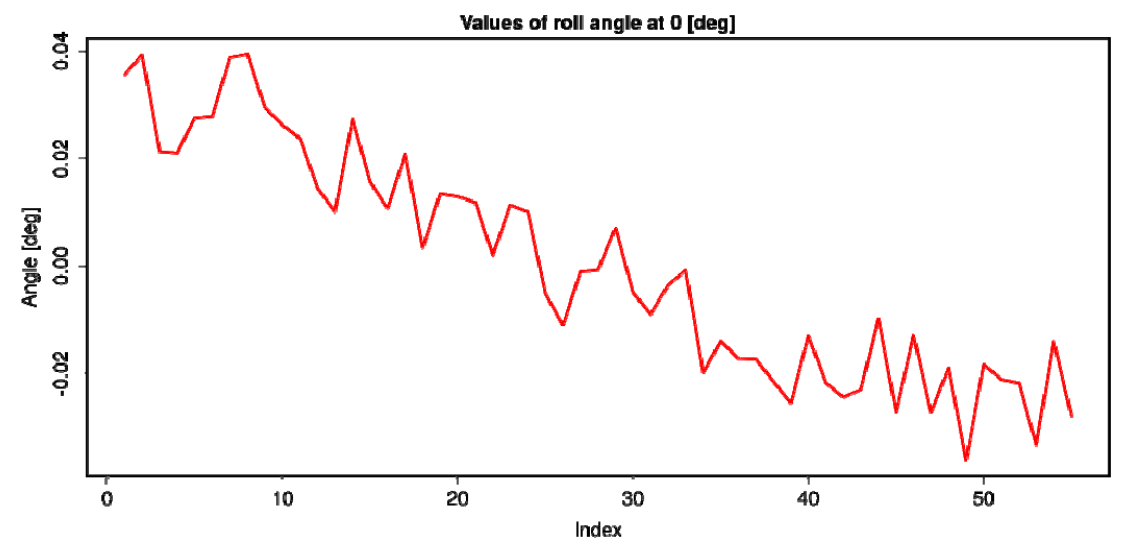

Fig. 13 Roll values calculated for $0^{\circ}$ tilt.

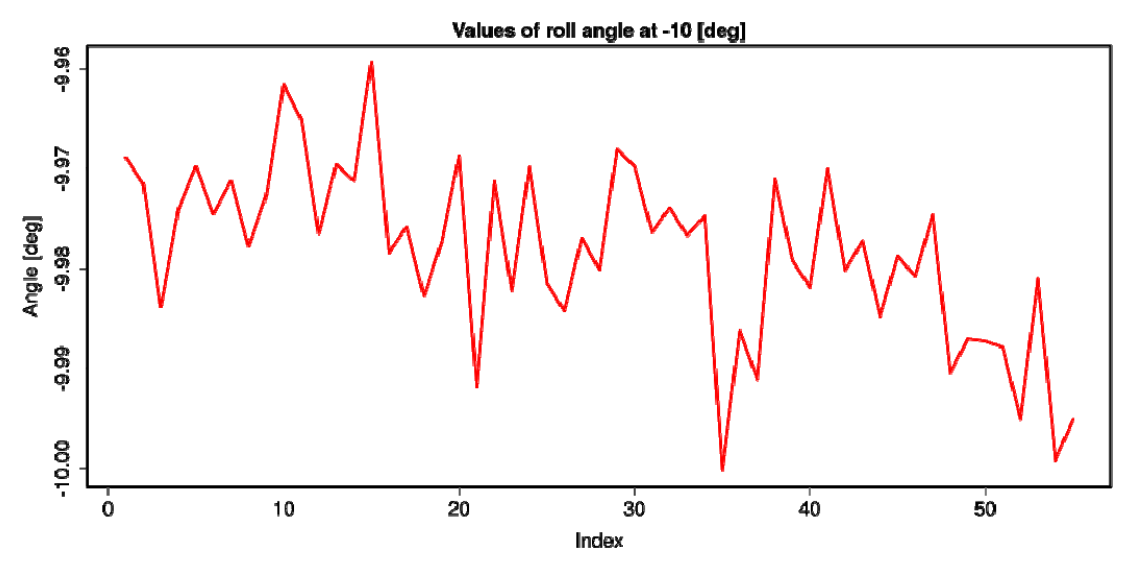

Fig. 14 Roll values calculated for $-10^{\circ}$ tilt.

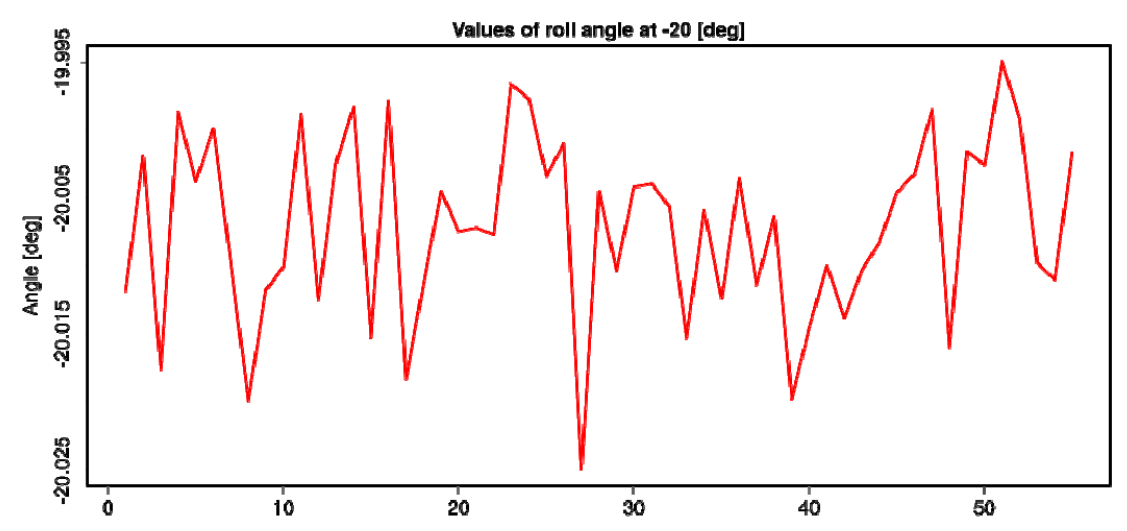

Fig. 15 Roll values calculated for $-20^{\circ}$ tilt.

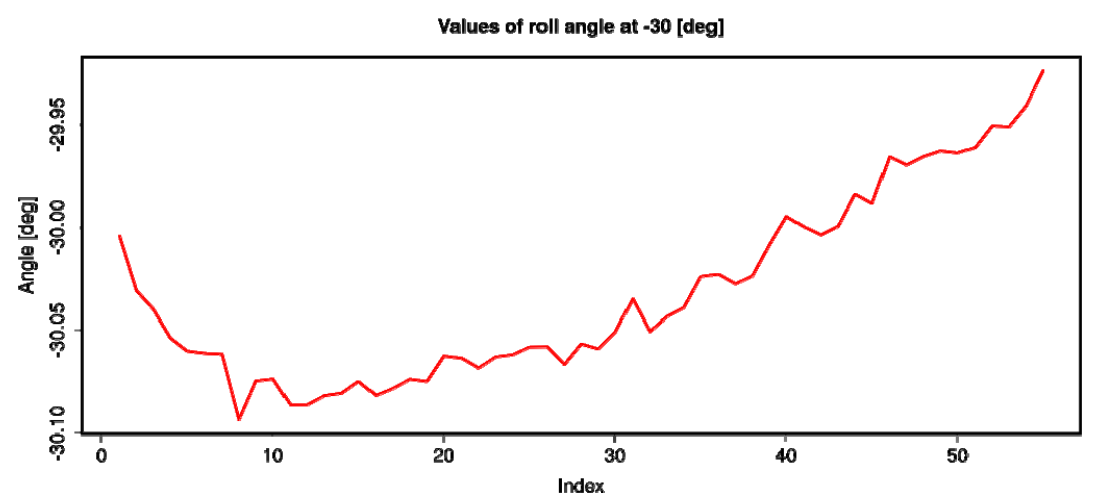

Fig. 16 Roll values calculated for $-30^{\circ}$ tilt. 
Table 3 Summary of conducted measurements.

\begin{tabular}{rrrrrc}
\hline Angle & mean & Min & Max & variance & $\begin{array}{c}\text { Standard } \\
\text { deviation }\end{array}$ \\
\hline $0^{\circ}$ & $0.000^{\circ}$ & $-0.036^{\circ}$ & $0.0394^{\circ}$ & $0.0000814^{\circ}$ & $0.0090^{\circ}$ \\
$-10^{\circ}$ & $-9.978^{\circ}$ & $-10.000^{\circ}$ & $-9.959^{\circ}$ & $0.0000799^{\circ}$ & $0.0089^{\circ}$ \\
$-20^{\circ}$ & $-20.010^{\circ}$ & $-20.020^{\circ}$ & $-19.993^{\circ}$ & $0.0000462^{\circ}$ & $0.0068^{\circ}$ \\
$-30^{\circ}$ & $-30.020^{\circ}$ & $-30.040^{\circ}$ & $-30.000^{\circ}$ & $0.0000765^{\circ}$ & $0.0087^{\circ}$ \\
\hline \multicolumn{7}{r}{ mean } & & $0.0000710^{\circ}$ & $0.0084^{\circ}$ \\
\hline
\end{tabular}

$43.2^{\circ}$. Therefore, installed gyro is not able to detect the rotation of the earth, because its value is smaller than the white noise affecting the measurement.

\section{CONCLUSIONS}

The accuracy of measurements made by ADIS 16354 was calculated, on the basis of conducted studies. The values of velocity random walk and bias stability for accelerometer installed within the unit were determined. The mean value of VRW was $0.25 \mathrm{~m} / \mathrm{s} / \sqrt{h}$ and bias stability $0.030 \mathrm{~m} / \mathrm{s}^{2}$. These results suggest that a medium quality accelerometer was installed within the examined IMU unit. Obtained accuracy of pitch and roll angles indicates that used device is sufficient for tilt compensation on measured accelerations. The values on angle random walk and gyro bias stability were determined as well. The mean value of ARW was $5.84 \% / \sqrt{h}$ and bias stability $0.013 \% \mathrm{~s}$. Calculated errors indicate that the quality of observations made by the gyroscope installed within ADIS unit is not sufficient for yaw angle determination. Therefore, it is suggested that the proposed set (ADIS $16354+$ LEA-6T), was accompanied by a magnetometer which allowed the determination of magnetic heading. Although the resulting error values may seem large, one must bear in mind that the device will constitute a part of an integrated navigation system. In systems of this type, results from the inertial navigation are supported by the measurements from GPS system with frequency $1 \mathrm{~Hz}$. Naturally the GPS signal may be lost in areas with low visibility of navigational satellites, but that situation can occur for no more than 30-60 s, even up to $360 \mathrm{~s}$, and does not cause a large degradation of accuracy in position determination. Obtained results demonstrate that the accuracy of ADIS 16354 is sufficient to use it as a part of integrated navigation system.

\section{REFERENCES}

Allan, D.: 1966, Statistics of Atomic Frequency Standards. In: Proc. of the IEEE, 54, 221-230.

Analog Devices. ADIS16354: High Precision Tri-Axial Inertial Sensor. http: www.analog.com.

Farrell, J.: 2008, Aided navigation GPS with high rate sensors, McGraw-Hill.
Foxlin, E.: 2005, Pedestrian tracking with shoe-mounted inertial sensors, IEEE Comput. Graph. Appl., 25(6), $38-46$.

Groves, D.: 2008, Principles of GNSS, inertial, and multisensory integrated navigation systems. Artech House, London.

Han, S., Wan, J. and Knight, N.: 2009, Using Allan variance to determine the calibration model of inertial sensors for GPS/INS integration. 6th International Symposium on Mobile Mapping Technology, Presidente Prudente, São Paulo, Brazil, July 21-24.

Noureldin, A., Karamat, T. and Georgy, J.: 2013, Fundamentals of inertial navigation, satellite-based positioning and their integration, Springer, Berlin.

Petkov, P. and Slavov, T.: 2010, Stochastic modeling of MEMS inertial sensors. Cybernetics and Information Technologies, 10, No 2, 31-40.

Stockwell, W.: Angle Random Walk. http://www.xbow.com.

Titterton, D.H. and Weston, J.L.: 2009, Strapdown Inertial Navigation Technology. American Institute of Aeronautics and Astronautics, Inc.

Wang, H. and Williams, T.: 2008, Strategic inertial navigation systems-high-accuracy inertially stabilized platforms for hostile environments. IEEE Control System Magazine, 28(1), 65-85. DOI: 10.1109/MCS.2007.910206

Woodman, O.: 2007, An introduction to inertial navigation. Technical Report No 696, Cambridge, United Kingdom.

Vukmirica, V., Trajkovski, I. and Asanović, N.: 2010, Two methods for the determination of inertial sensor parameters. Scientific Technical Review, 60, No. 3-4, 27-33. 